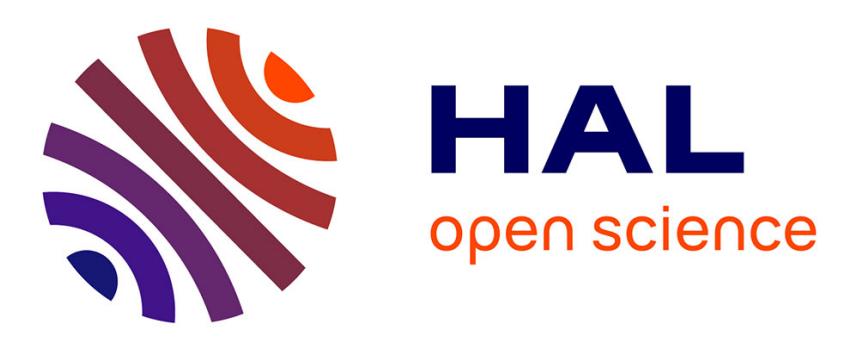

\title{
Tailored structuring of functionalized silsesquioxanes in a one-step approach
}

Robert Winkler, S Pellet-Rostaing, Guilhem Arrachart

\section{To cite this version:}

Robert Winkler, S Pellet-Rostaing, Guilhem Arrachart. Tailored structuring of functionalized silsesquioxanes in a one-step approach. Materials Chemistry Frontiers, 2021, 5 (5), pp.2328-2337. 10.1039/d0qm01060j . hal-03395190

\section{HAL Id: hal-03395190 \\ https://hal.science/hal-03395190}

Submitted on 22 Oct 2021

HAL is a multi-disciplinary open access archive for the deposit and dissemination of scientific research documents, whether they are published or not. The documents may come from teaching and research institutions in France or abroad, or from public or private research centers.
L'archive ouverte pluridisciplinaire HAL, est destinée au dépôt et à la diffusion de documents scientifiques de niveau recherche, publiés ou non, émanant des établissements d'enseignement et de recherche français ou étrangers, des laboratoires publics ou privés. 


\title{
Tailored structuring of functionalized silsesquioxanes in an one-step approach
}

\author{
Robert Winkler,* Stéphane Pellet-Rostaing and Guilhem Arrachart* \\ ICSM, Univ Montpellier, CEA, CNRS, ENSCM, Marcoule, France. \\ E-mail: guilhem.arrachart@cea.fr
}

\begin{abstract}
The silica hybrid materials prepared from tailor-made precursors with different headgroups in several experimental conditions (acid or base catalysis in water or THF) were studied in order to identify the structure directing mechanisms. By infrared spectroscopy and small-angle X-ray scattering and thermogravimetric analysis, it was found that the molecular interactions such as Coulomb interactions, vdW forces, hydrogen bonds and sterical factors that are strongly influenced by the experimental conditions, orient the main structural features. Secondarily, the chemically directed connectivity of the growing Si-O-Si network nuances the final structure. Finally, on heating to $130{ }^{\circ} \mathrm{C}$, lamellar structures remained stable whereas the deformation of hexagonal and cubic stuctures shows metastability. The results can be regarded as an interaction tool box for "all-in-one" material chemists.
\end{abstract}

\section{Introduction}

Inorganic-organic hybrid materials advantageously combine the properties of modulable organic moieties and robust inorganic matrices. ${ }^{1}$ To successfully tailor physical and chemical properties of the material, the efficient incorporation of the required organic moiety and the controlled formation of the inorganic matrix are crucial. To this end, the sol-gel process of silica materials has become a considerable tool due to the, often, mild reaction conditions and convenient reaction kinetics. Adapting the nature of the precursor and the reaction conditions (solvent, temperature, catalyst...) the synthesis of a wide variety of silica hybrid materials (SHMs) is possible. Classically, it is done in a two- or multi-step procedure. In most cases, first, the inorganic matrix is created by applying the principles of soft matter structuring to control porosity and wall properties. In a second step, the structure directing template molecule is removed by thermal or chemical treatment or washing. Finally, the organic function is introduced by post-functionalization. Although well-studied and, thus, effective, this approach has several disadvantages. The synthesis of the inorganic matrix is associated to a large production of waste like the template surfactant and the washing solutions. This includes the thermic cost of template removal by combustion. Despite the fact that these disadvantages might be outsourced by buying a commercially available product, for example, SBA-15, their negative impact persists and the client loses the access to a tailor-made product. Moreover, during the post-grafting step, several problems can occur such as the pore clogging due to the limited accessibility of molecules to the pore wall surface ${ }^{2-4}$, the inhomogeneity of the grafting ${ }^{4,5}$ and a wall-facing functional group orientation ${ }^{6}$. An alternative approach for the synthesis of tailor-made SHMs is a unimolecular "all-in-one" approach. It consists in combining the required components - silica precursor, template molecule, organic moiety - into one single precursor molecule - the organosilane precursor - that forms the desired material under the right reaction conditions. Ultimately, this approach has the potential to eliminate all of the mentioned 
disadvantages of the classical multistep approach. No sacrificial template molecule is used that needs subsequent removal. The structuring is piloted by the interactions between the precursors that are conditioned by the reaction parameters. The porosity of such a material will not be clogged in a postfunctionalization step since the organic moiety is already incorporated. Hence, inhomogeneous grafting and wall-facing groups are avoided.

In the last decade, this concept was explored to form a variety of original bridged silsesquioxanes ${ }^{7-17}$ by playing with several parameters of which the interactions such as $\pi$-stacking, hydrophobic interactions or hydrogen bonding. Following the same unimolecular all-in-one approach, the aim of this study is to work towards the generalization of the effects of different reaction parameters on the resulting material structure. To this end, several home-made organosilane precursors ${ }^{18}$ (see Figure 1 ) with amine or alkyl groups and functional groups effective at rare earth extraction ${ }^{19,20}$ were polymerized under different reaction conditions and, then, thermally treated (see Figure 2).

The reactions to form the silsesquioxanes were carried out in aqueous solution or in tetrahydrofuran (THF) under acidic or basic catalysis. More information is provided in the ESI or in literature. ${ }^{20}$

Using thermogravimetric analysis (TGA), infrared spectroscopy (FT-IR) and small angle X-ray scattering (SAXS), three different factors driving the properties of the sol and the final materials were considered: (i) the silica condensation mechanism of the inorganic part of the precursors as a function of $\mathrm{pH}$ and the solvent; (ii) the organic functional group of the precursors and their steric and electronic properties and (iii) the interactions between the organosilanes driven by the functional group, the $\mathrm{pH}$ and the solvent.

a)

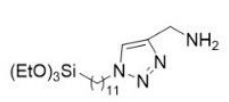

P1

d)

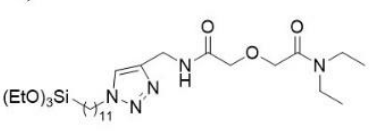

P4 b)

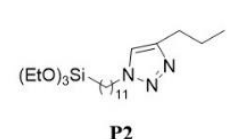

c)

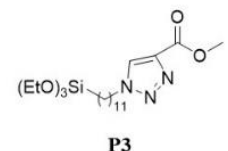

P3

e)

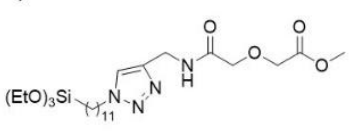

P5

Figure 1. Organosilane precursor molecules used in the present work.

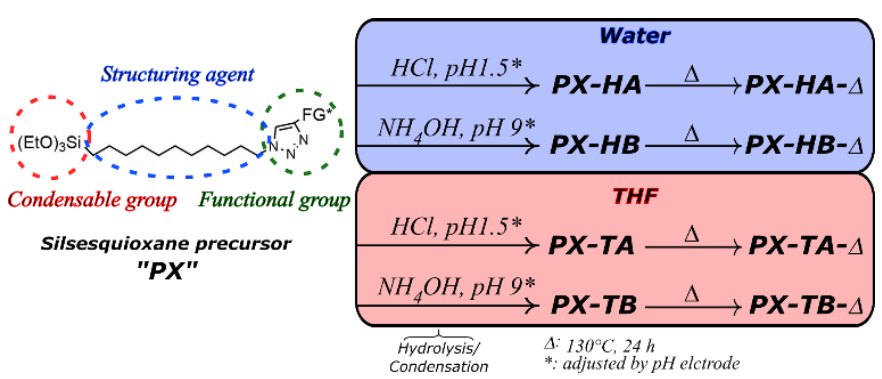

Figure 2. Overview of the silsesquioxane precursors, the experimental conditions used to prepare the SHMs with their references where $\mathrm{X}$ is $1,2,3,4$ or 5 depending on the precursor. 


\section{Results and Discussion}

This section is separated into three parts that thoroughly investigate the material structure in a hierarchical manner. Beginning with the composition and the molecular structure, going to the supramolecular organization, this section ends with the morphology at the mesoscale. Afterwards, the results are discussed along the lines of the three mentioned factors in order to propose the material formation mechanisms.

\section{Composition and molecular structure}

The material composition was investigated using FT-IR and TGA. Figure shows the FT-IR spectra of the resulting materials before (black) and after thermal treatment (coloured). Some data was already published in another context. ${ }^{21}$ In all spectra, characteristic peaks of the present SHMs are found $\left(v_{1}-v_{6}\right)$. They are common to all materials such as the $\mathrm{C}-\mathrm{H}$ stretching mode of the triazole moiety $\left(\sim 3130 \mathrm{~cm}^{-1}\right.$, $\left.\mathrm{v}_{1}\right)$, the asymmetric $\left(\sim 2920 \mathrm{~cm}^{-1}, \mathrm{v}_{2}\right)$ and symmetric $\left(\sim 2850 \mathrm{~cm}^{-1}, \mathrm{v}_{3}\right) \mathrm{CH}_{2}$ stretching modes, the $\mathrm{C}=\mathrm{C}$ stretching mode of the triazole moiety $\left(\sim 1460 \mathrm{~cm}^{-1}, \mathrm{v}_{4}\right)$ that is split for $\mathrm{P} 3$ due to conjugation with $\mathrm{C}=\mathrm{O}$ $\left(v_{4 a}, v_{4 b}\right)$, the Si- $\mathrm{CH}_{2}$ - stretching mode $\left(\sim 1210 \mathrm{~cm}^{-1}, v_{5}\right)$, and the various Si-OH stretching modes ( 1020$\left.930 \mathrm{~cm}^{-1}, \mathrm{v}_{6}\right){ }^{22-24}$ These peaks confirm the retention of the $\mathrm{Si}-\mathrm{C}$ bond and the triazole moiety. For P3$\mathrm{TB} /-\Delta$, the broad peaks between 3400 and $2400 \mathrm{~cm}^{-1}$ around the $\mathrm{C}=0$ mode, and at $1400 \mathrm{~cm}^{-1}$ reveal the formation $\mathrm{NH}_{4} \mathrm{Cl}$ due to an unintended addition $\mathrm{HCl}$ to the sol that was not properly removed. In the following, the results data for this material are, nonetheless, presented but not discussed. After the thermal treatment, an expected attenuation (sometimes even disappearance) of the $\mathrm{Si}-\mathrm{OH}$ peaks is observed.

a)

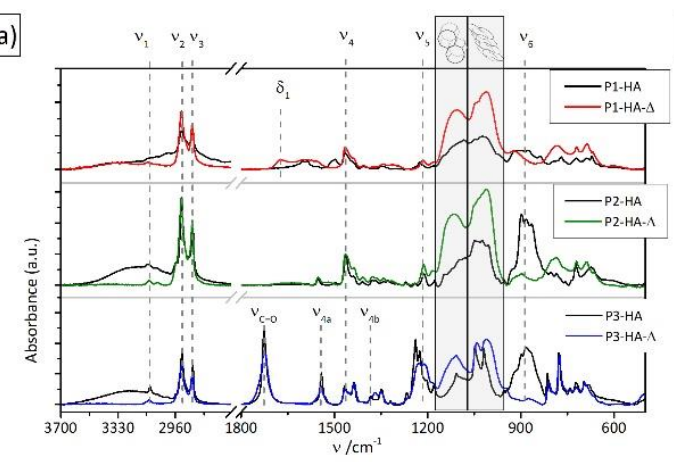

c)

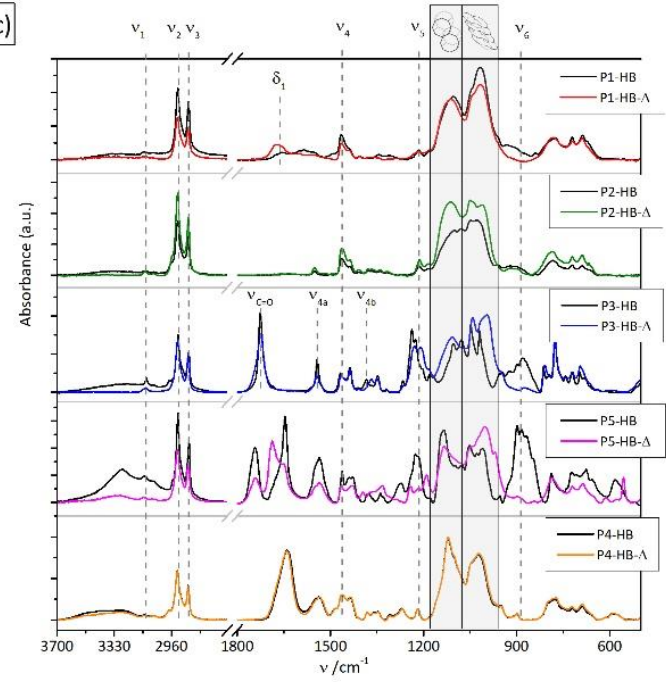

b)

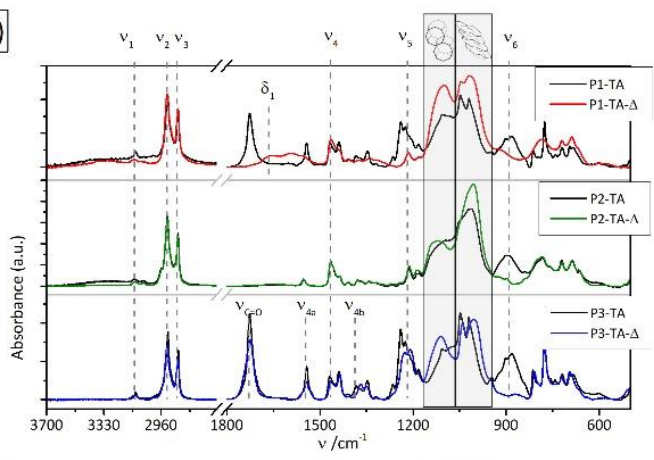

d)

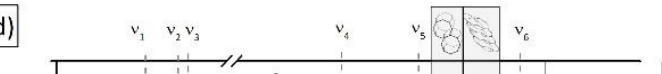

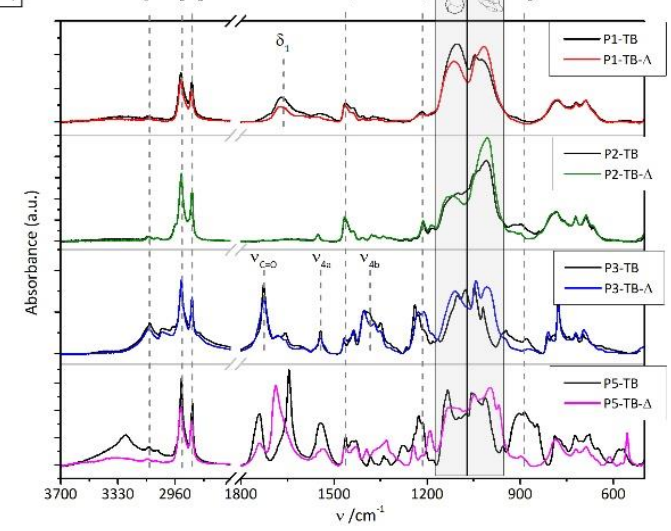

Figure 3. FT-IR spectra of the obtained materials before (PX-YY) and after thermal treatment (PX-YY- $\Delta$ ). The left-hand side (a) and c)) show the materials prepared from aqueous solution while the right-hand side (b) and d)) shows the materials prepared in THF.

Catalysis was acidic for the materials shown in a) and b) and basic for the materials shown in c) and d). The grey insets highlight the regions were the characteristic signals for ladder or cage-like supramolecular structures are found. Some of the data was already published in a different context. ${ }^{20}$ 
This process indicates the advancing condensation of the Si-O-Si network. Consistently, the intensity of the peaks in the region between 1200 and $1000 \mathrm{~cm}^{-1}$ that are assigned to the Si-O-Si stretching modes increases. Furthermore, some precursor specific peaks are observed corresponding to the $\mathrm{N}-\mathrm{H}$ bending mode of the primary amine of $\mathrm{P} 1\left(\sim 1660 \mathrm{~cm}^{-1}, \delta_{1}\right)$ and the $\mathrm{C}=\mathrm{O}$ stretching modes for P3, P4 and P5 in the region comprised between 1800 and $1600 \mathrm{~cm}^{-1}$. For the latter, the different carbonyl functions are identified. P3 materials show a single peak that is assigned to the methyl ester and for P4 the large peak is composed of the $3^{\circ}$ and $2^{\circ}$ amide. No change is observed after thermal treatment. For P5 the situation is more complex. A zoom on the region (see Figure S1 ESI) reveals three different peaks. The ester peak at $\sim 1740 \mathrm{~cm}^{-1}$ shows an intensity decrease after the thermal treatment. The other two peaks are both attributed to the $2^{\circ}$ amide. The reason for this splitting may be the formation of hydrogen bonds that typically lead to a red-shift. For the fresh material, this leads to a shifted peak at $1650 \mathrm{~cm}^{-1} \mathrm{lt}$ is probably overlapped by the bending mode of residual water. After thermal treatment, this peaks intensity strongly decreases for the benefit of the free $2^{\circ}$ amide peak $\left(1690 \mathrm{~cm}^{-1}\right)$.

The region between 3600 and $2800 \mathrm{~cm}^{-1}$ contains the merged peaks of several X-H $(X=H-O, N, S i-O)$ bonds. For all materials, the intensity of this region decreases after the thermal treatment due to the evaporation of residual water and the condensation of free $\mathrm{Si}-\mathrm{OH}$ bonds. In this region, a peak of low intensity residual intensity is observed in the cases of P1, P4 and P5 due to the $\mathrm{N}-\mathrm{H}$ bonds. It should be noted that for the acid catalysed fresh materials from P1 (P1-HA, P1-TA) a particular peak between 3000 and $2800 \mathrm{~cm}^{-1}$ is observed. This peak disappears after the washing with a basic solution. The simultaneous absence of $\delta_{1}$ indicates the formation of a primary ammonium group. Another particular feature is the sharpness of the broad peak for the fresh P5 materials at $3280 \mathrm{~cm}^{-1}$ that indicates water hydrogen bonding. ${ }^{25-27}$ This supports the presence of hydrogen bonding in the fresh materials $\mathrm{P} 5-\mathrm{HB}$ and P5-TB between residual water and the $2^{\circ}$ amide.

TGA results in Figure were used to assess the Si-O-Si network condensation and to calculate the molecular weight of the final material. For all materials, the weight loss occurs in consecutive but indistinguishable steps beginning around 200 to $300{ }^{\circ} \mathrm{C}$ which is characteristic for the decomposition of the triazole group. ${ }^{28}$ The absence of weight loss at lower temperatures which would be typical for water evaporation or $\mathrm{Si}-\mathrm{OH}$ condensation, confirms the effective network consolidation. After $600{ }^{\circ} \mathrm{C}$, the residual mass remains constant indicating the complete decomposition of the organic part. FT-IR investigations after the measurement (see Figure S2 ESI) show that the material heated to $1000{ }^{\circ} \mathrm{C}$ is ssentially $\mathrm{SiO}_{2}{ }^{24}$

Exploiting the molar equivalence of silicon in the residual and the thermally treated material using (1)

$$
M_{T G A}=100 * \frac{M_{\mathrm{SiO}_{2}}}{W_{700^{\circ} \mathrm{C}}}=316 \pm 20 \mathrm{~g} \mathrm{~mol}^{-1}
$$

where MTGA is the molecular weight of the material as determined by TGA, Msio2 the molecular weight of silica $\left(60.09 \mathrm{~g} \mathrm{~mol}^{-1}\right)$ and $W_{700}{ }^{\circ} \mathrm{C}$ the residual mass percentage at $700{ }^{\circ} \mathrm{C}$, the molecular weight was calculated. The results are shown in Table 1.

For all materials, the calculated molecular weights are in good agreement with the theoretical data. A small tendency towards overestimation is noticed which might be due to incomplete condensation of the Si-OH groups.

Concluding the first part, the molecular structure of the synthesized silsesquioxanes was confirmed for all obtained materials by the identification of all relevant peaks in the FT-IR spectra. Concerning the composition, an incomplete condensation is observed for the fresh materials by the presence of the $\mathrm{Si}$ $\mathrm{OH}$ peak. After thermal treatment, the attenuation of this peak indicates the Si-O-Si network condensation. Moreover, the molecular weights obtained by TGA confirm this observation. Finally, some particularities are observed in the FT-IR spectra that are discussed in a later context. 
a)
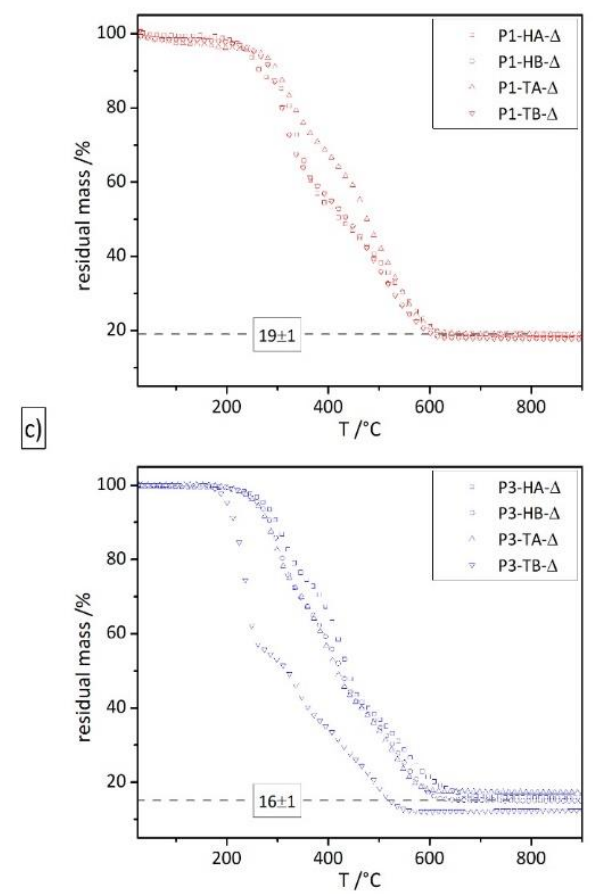

b)
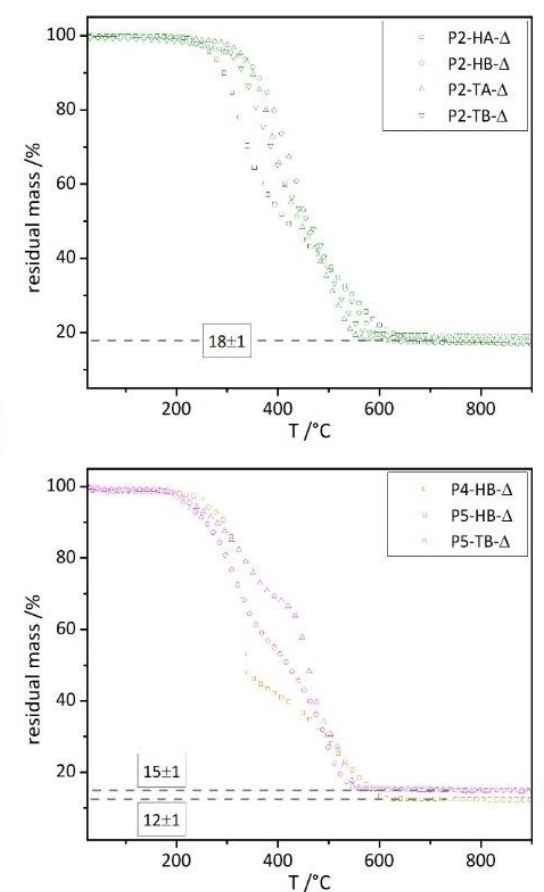

Figure 4. TGA weight loss curves for the obtained materials after thermal treatment sorted by precursor. The dashed line highlights the final residual mass.

Table 1. Residual masses and the corresponding molecular weights of the obtained materials after thermal treatment as determined by TGA. The last column shows the calculated theoretical molecular weight of the completely condensed precursor.

\begin{tabular}{|c|c|c|c|c|c|c|}
\hline Material & $w 700^{\circ} \mathrm{C}(w \%)$ & $\mathrm{M}_{\mathrm{TGA}}\left(\mathrm{g} \mathrm{mol}^{-1}\right)$ & Material & $w 700^{\circ} \mathrm{C}(w \%)$ & $\mathrm{M}_{\mathrm{TGA}}(\mathrm{g} \mathrm{mol}-1)$ & $\mathrm{M}_{\text {theo }}\left(\mathrm{g} \mathrm{mol}^{-1}\right)$ \\
\hline P1-HA- $\Delta$ & $19 \pm 1$ & $316 \pm 20$ & P1-TA- $\Delta$ & $19 \pm 1$ & $316 \pm 20$ & \multirow{2}{*}{303} \\
\hline P1-HB- $\Delta$ & $20 \pm 1$ & $300 \pm 20$ & P1-HB- $\Delta$ & $18 \pm 1$ & $333 \pm 20$ & \\
\hline P2-HA- $\triangle$ & $17.5 \pm 1$ & $343 \pm 20$ & P2-TA- $\Delta$ & $18 \pm 1$ & $334 \pm 20$ & \multirow{2}{*}{317} \\
\hline P2-HB- $\Delta$ & $17.5 \pm 1$ & $343 \pm 20$ & P2-TB- $\Delta$ & $19 \pm 1$ & $317 \pm 17$ & \\
\hline P3-HA- $\triangle$ & $16 \pm 1$ & $376 \pm 25$ & P3-TA- $\triangle$ & $17.5 \pm 1$ & $342 \pm 25$ & \multirow{2}{*}{376} \\
\hline P3-HB- $\Delta$ & $16 \pm 1$ & $376 \pm 25$ & P3-TB- $\Delta$ & & & \\
\hline P4-HB- $\triangle$ & $12.5 \pm 1$ & $481 \pm 30$ & & & & 475 \\
\hline P5-HB- $\Delta$ & $15 \pm 1$ & $401 \pm 30$ & P5-TB- $\Delta$ & $15 \pm 1$ & $401 \pm 30$ & 432 \\
\hline
\end{tabular}

\section{Supramolecular organization}

FT-IR data (see Figure) were used to determine the arrangement of the monomers inside the silsesquioxanes as described in literature. ${ }^{29-32}$

First, the position of the asymmetric $\mathrm{CH}_{2}$ stretching mode $\left(\mathrm{v}_{2}\right)$ and the intensity ratio of the asymmetric to the symmetric mode $R_{\text {alkyl }}=I\left(v_{2}\right) / I\left(v_{3}\right)$ gives information on the alkyl chain conformation. Second, the two broad peaks between 1200 and $1000 \mathrm{~cm}^{-1}$ give information on the geometry of the Si-O-Si bridges that relates to the overall structure of the inorganic phase. In silsesquioxanes, they are typically assigned to cage-like motifs $\left(\mathrm{Pc}_{\mathrm{c}} \sim 1110 \mathrm{~cm}^{-1}\right)$ and open chain or ladder-like motifs $\left.\left(\mathrm{PL}_{\mathrm{L}} \sim 1020 \mathrm{~cm}^{-1}\right)\right)^{31,33}$ Their ratio $R_{\text {Siosi }}=I\left(P_{C}\right) / I\left(P_{L}\right)$ indicates the predominant motif. The values for the materials prepared from P3 need to be taken with care and those for P4 and P5 were not calculated because of the overlay with carbonyl related peaks. The obtained values are shown in Table 2. 
Table 2. Data of the FT-IR analyses concerning the supramolecular organisation. The columns list the position of the asymmetric $\mathrm{CH}_{2}$ stretching mode, the ratio $\mathrm{R}_{\text {alkyl }}$ and the ratio $R_{\text {siosi. }}$

\begin{tabular}{|c|c|c|c|c|c|c|c|}
\hline Material & Pos $v_{2}\left(\mathrm{~cm}^{-1}\right)$ & Ralkyl & Rsiosi & Material & Pos $v_{2}\left(\mathrm{~cm}^{-1}\right)$ & Ralkyl & $R_{\text {siosi }}$ \\
\hline P1-HA- $\Delta$ & 2921 & 1.27 & 0.77 & P1-TA- $\Delta$ & 2921 & 1.25 & 0.9 \\
\hline P1-HB- $\triangle$ & 2921 & 1.3 & 0.81 & P1-TB- $\Delta$ & 2921 & 1.26 & 1.18 \\
\hline P2-HA- $\Delta$ & 2920 & 1.34 & 0.74 & P2-TA- $\Delta$ & 2919 & 1.37 & 0.44 \\
\hline P2-HB- $\Delta$ & 2920 & 1.4 & 0.98 & P2-TB- $\Delta$ & 2919 & 1.35 & 0.43 \\
\hline P3-HA- $\Delta$ & 2920 & 1.34 & 0.75 & P3-TA- $\Delta$ & 2921 & 1.35 & 0.79 \\
\hline P3-HB- $\Delta$ & 2920 & 1.36 & 0.71 & P3-TB- $\Delta$ & 2920 & 1.31 & 0.94 \\
\hline P4-HB- $\Delta$ & 2921 & 1.5 & & & & & \\
\hline P5-HB- $\Delta$ & 2918 & 1.34 & & P5-TB- $\Delta$ & 2919 & 1.34 & \\
\hline
\end{tabular}

All materials show an extended all-trans configuration of the alkyl chains $\left(\operatorname{Pos}\left(\mathrm{V}_{2}\right) \approx 2920 \mathrm{~cm}^{-1}\right.$, $1.2<$ Ralkyl $_{1} 1.4$ ) except one. For P4-HB- $\Delta$ the ratio is superior indicating a (partially) collapsed alkyl chain. Concerning the $\mathrm{Si}-\mathrm{O}-\mathrm{Si}$ geometry, the tendencies vary between the precursors. For the materials synthesized from P1, the synthesis in THF yields higher shares of cage-like motifs than synthesis in aqueous conditions. In both cases base catalysis yields the highest share of cage-like motifs. For P2, synthesis in THF leads to overall higher shares of ladder-like motifs without a clear distinction of acid or base catalysis. Under aqueous conditions, the share of cage-like motifs is higher, especially with base catalysis. For P3-HA- $\Delta$, P3-HB- $\Delta$ and P3-TA- $\Delta$, a dominance of ladder-like structures is observed. The share of cage-like motifs is slightly higher for P3-TB- $\Delta$.

Major influences on the ratio of cage- to ladder-like motifs are the precursor and the water concentration and the $\mathrm{pH}$ during the sol-gel process. ${ }^{34-36}$ Under basic conditions, coherent with the known strong ramification, a tendency to cage-like motifs is observed. The degree depends on the solvent and the precursor. For P2, the influence of precursor solubility and water concentration are coherent with literature. The higher solubility of the hydrophobic precursor in THF than in water and the lower water concentration in THF lead to a higher share of ladder-like motifs. For the precursors P3 and P1 the difference is less obvious because of the more complicated molecular structure. Precursor-specific effects arise from the interactions between the functional groups and the solvent. This is complicated further by the protonation and the bola-amphiphilic character in some cases. A more thorough discussion is given ulteriorly.

\section{Mesoscale structuring}

Lastly, the morphology at the mesoscale is characterized. To this end, SAXS as performed. The results are shown in Figure .

For all materials, at $\mathrm{q}<1 \mathrm{~nm}^{-1}$, a slope proportional to $\mathrm{q}^{-\alpha}$ where $\alpha$ is comprised between 3 and 4 is observed. This indicates solid materials varying between smooth $(\alpha=4)$ and rough $(\alpha=3)$ surfaces. At higher q, several Bragg peaks can be observed, that allow the assignment of different mesophases. For the materials synthesized from P1, the assignment is possible for the materials P1-HB/- $\Delta$ and P1-TA/- $\Delta$. The equidistant positions of the Bragg peaks $\left(q_{\times 00}\right)$ indicate a lamellar structure. After the thermal treatment, the Bragg peaks shift to higher $q$ and a repeat unit length of $3.6 \mathrm{~nm}$ is found that corresponds to two extended precursor molecules. ${ }^{10}$ The shift indicates a material contraction and is more distinct for the materials synthesized under basic conditions. A possible explanation is the release of $\mathrm{CO}_{2}$ due to the thermal decomposition of carbamates that are formed in situ, as reported in an earlier study. ${ }^{10}$ For P1-TA- $\Delta$ the repeat unit length is confirmed by TEM (see Figure S3 a and b) ESI). For the other materials, a structure assignment is ambiguous. For P1-TB/- $\Delta$ the presence of two peak no structure can be identified which is confirmed by TEM (see Figure S3 c) ESI). For P1-HA/- $\Delta$, a lamellar structure is suspected but the peak width and the absence of higher order peaks impedes a conclusion. 
a)

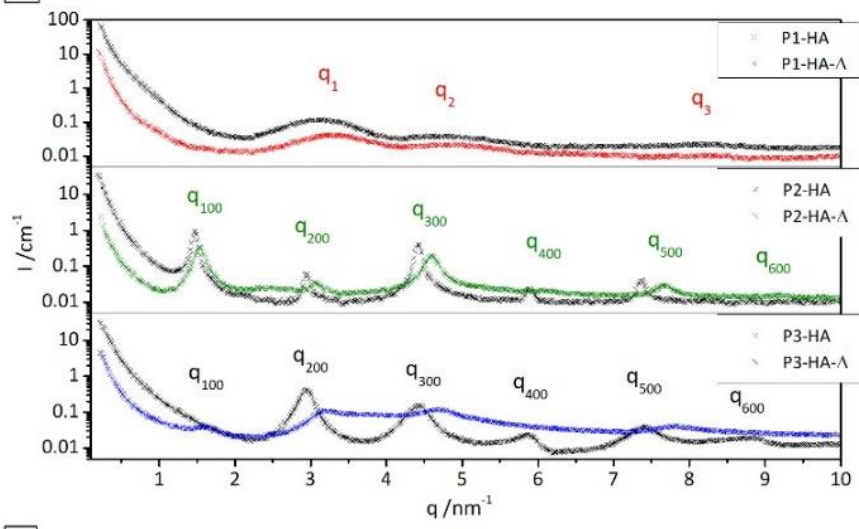

c)

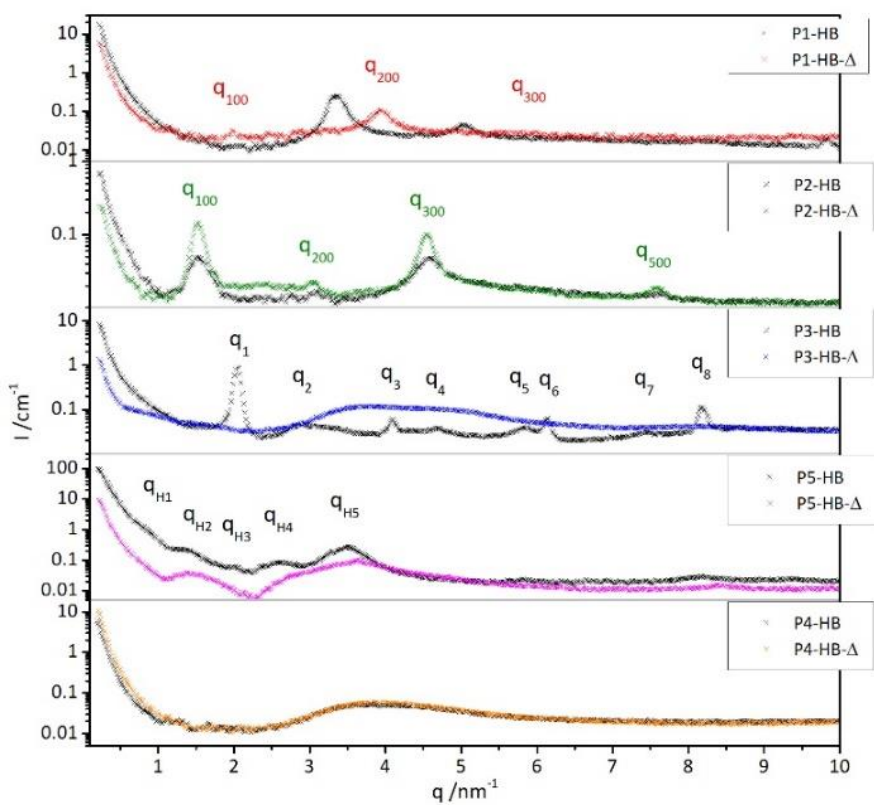

b)

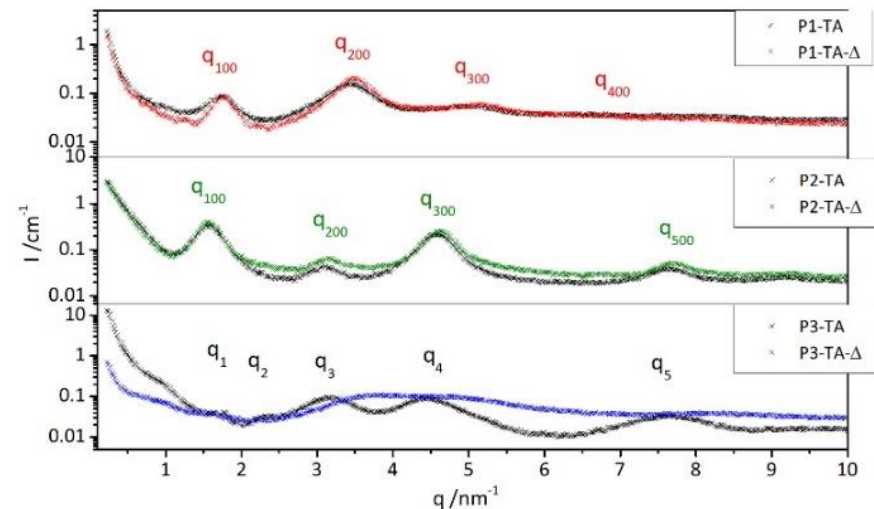

d)

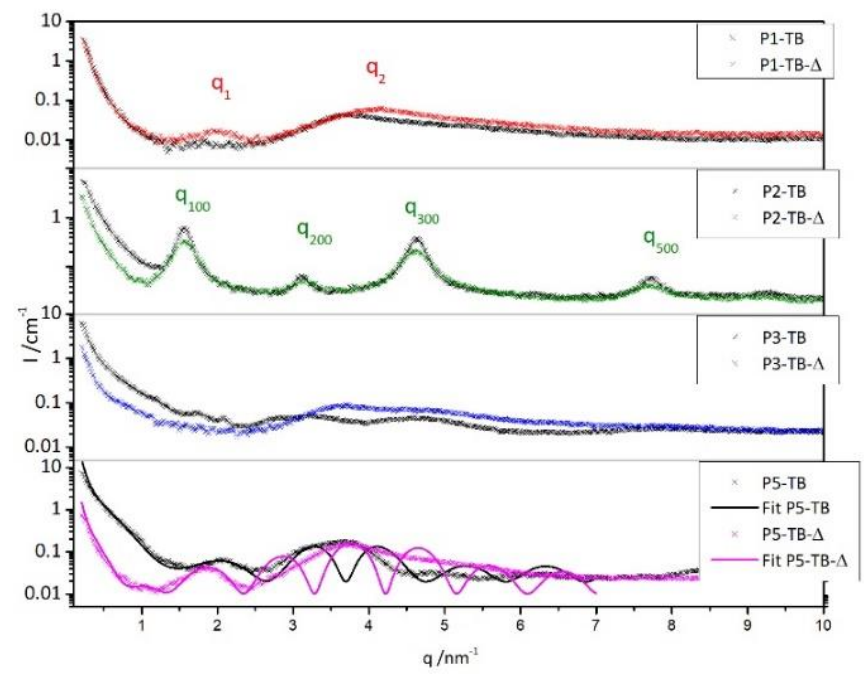

Figure 5. SAXS patterns of the obtained materials before (PX-YY) and after thermal treatment (PX-YY- $\Delta$ ). The left-hand side (a) and c)) show the materials prepared from aqueous solution while the right-hand side (b) and d)) shows the materials prepared in THF. Catalysis was acidic for the materials shown in a) and b) and basic for the materials shown in c) and d). The highlighted $q$ values show Bragg-peaks that indicate the mesostructure as discussed in the text. The colour of these highlights indicates their assignment to the untreated (black) or the thermally treated samples (coloured).

For all P2-materials, the Bragg peaks allow the assignment of a lamellar structure. The increasing peak sharpness and the visibility of higher order peaks indicate increasing lamellar characteristics in the order $\mathrm{P} 2-\mathrm{TA} \approx \mathrm{P} 2-\mathrm{TB}<\mathrm{P} 2-\mathrm{HB}<\mathrm{P} 2-\mathrm{HA}$. For these materials, a peak attenuation for even-order peaks is observed that is due to a particularity in the scattering length density (SLD) distribution. ${ }^{37,38} \mathrm{After}$ thermal treatment, some peak smoothing is observed and a loss of long-range can be concluded. An exception is the material P1-HB where the intensity of peak $\mathrm{q}_{100}$ increases. This is explained by the high share of cagelike motifs. Indeed, these motifs act as defects in the lamellar structure. Upon further condensation during the thermal treatment, their SLD increases and thus the peak intensity. In the other materials, less cage-like motifs are observed by FT-IR rendering the effect invisible. For the materials synthesized from P3, the larger repertory of structures is found. Thanks to the presence of high-order Bragg peaks a lamellar structure for P3-HA, a D-bicontinous mesophase in a cubic lattice for P3-HB ${ }^{39}$, and a 2D hexagonal structuring are concluded. After thermal treatment, $\mathrm{P} 3-\mathrm{HA}-\Delta$ shows a contraction to a repeat unit length of $4 \mathrm{~nm}$ that corresponds to two extended precursor molecules. At the same time, some peak smoothing is observed that indicates the loss of long-range order. For the other materials, a significant loss of order is observed that is categorized as amorphization. 
For $\mathrm{P} 4-\mathrm{HB} /-\Delta$, a single large peak at $\sim 4 \mathrm{~nm}^{-1}$ is observed. The absence of further peaks indicates an amorphous structure. For the materials prepared from $\mathrm{P} 5$, the interpretation of the scattering patterns is not straightforward due to the complexity of the SLD profile. For P5-HB/- $\Delta$, the Bragg peaks noted $q_{H 1}$ to $q_{H 5}$ are at positions that are characteristic of a $2 \mathrm{D}$ hexagonal structure. The first peak at $q \approx 1 \mathrm{~nm}{ }^{-1}(6-$ $7 \mathrm{~nm}$ real space) is hard to distinguish due to its position on the slope. After the thermal treatment, the broadening peaks indicate increasing disorder. Finally, for P5-TB/- $\Delta$, a qualitative fit was done in order to interpret the patterns (see Figure 6). This simple fit simulates a lamellar structure of condensed precursor double layers. It does not claim a remodelling of the exact structure but serves as a guide to identify at least the second order peak $\left(q_{200}\right)$ and its position using reasonable SLDs and SLD changes. The peak shift indicates a dilation of the lamellar structure from 6 to $6.7 \mathrm{~nm}$ after the thermal treatment. Knowing from FT-IR that the alkyl chains are in an extended configuration, this corresponds to a change from intercalated to end-to-end arrangement of the lamellae. On a final note, it is mentioned that, both, P5-HB and P5-TB show several Bragg peaks in the WAXS region ( $q>10 \mathrm{~nm}^{-1}$ ) of the pattern (see Figure S4 ESI). They are more pronounced for P5-TB but disappear for both materials after the thermal treatment.

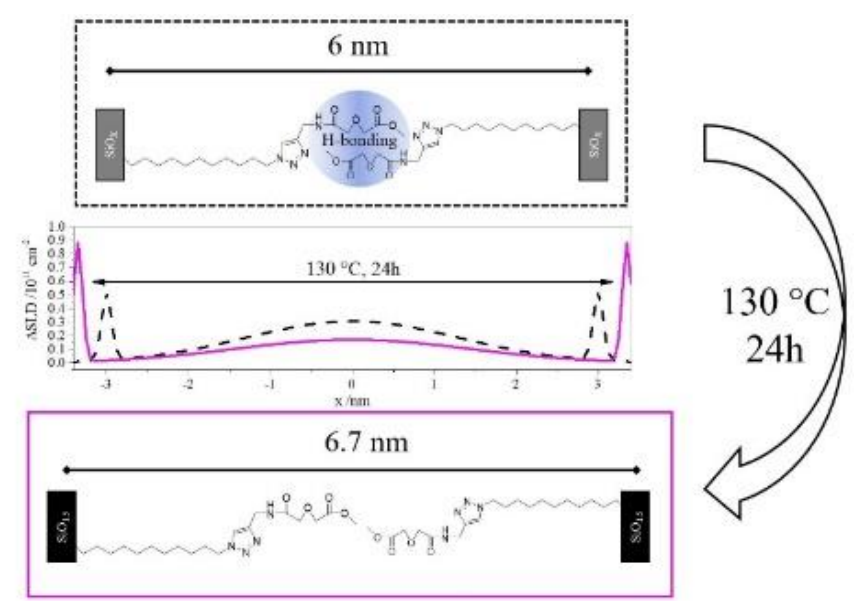

Figure 6. Scheme of the modelled evolution of material P5-TB upon thermal treatment. The centre figure is calculated using the fit parameters that produced the fits in Figure c).

\section{Discussion}

Regarding the obtained results, several SHM formation mechanisms are proposed taking the factors introduced earlier into account. They are (i) the silica condensation mechanism depending on $\mathrm{pH}$ and solvent, (ii) the organic functional group and its steric and electronic properties, and (iii) the interactions between the precursors driven by their molecular structure, the $\mathrm{pH}$ and the solvent. An overview of the results is shown in Figure 7.

In the case of P1, the share of ladder- or cage-like motifs is mostly directed by the silica condensation mechanism. Whatever the solvent, base catalysis leads to a higher share of cage-like motifs than acid catalysis. Intermolecular coulomb interactions could come into play in a mesoscale structuredetermining mechanism: The lamellar character of the materials is most pronounced for P1-TA/- $\Delta$ and $\mathrm{P} 1-\mathrm{HB}$ where the latter and the subsequent amorphization is largely explained by a mechanism described in literature. ${ }^{10}$ For $\mathrm{P} 1-\mathrm{TA} /-\Delta$, the proposed mechanism is organised by the charge-forming protonation of the amine group. Indeed, the acid induced protonation creates a charged ammonium headgroup that, in THF, tends to form counter-ion pairs. A hydrated, hydrophilic pseudophase is thus created with several of the precursor molecules. 


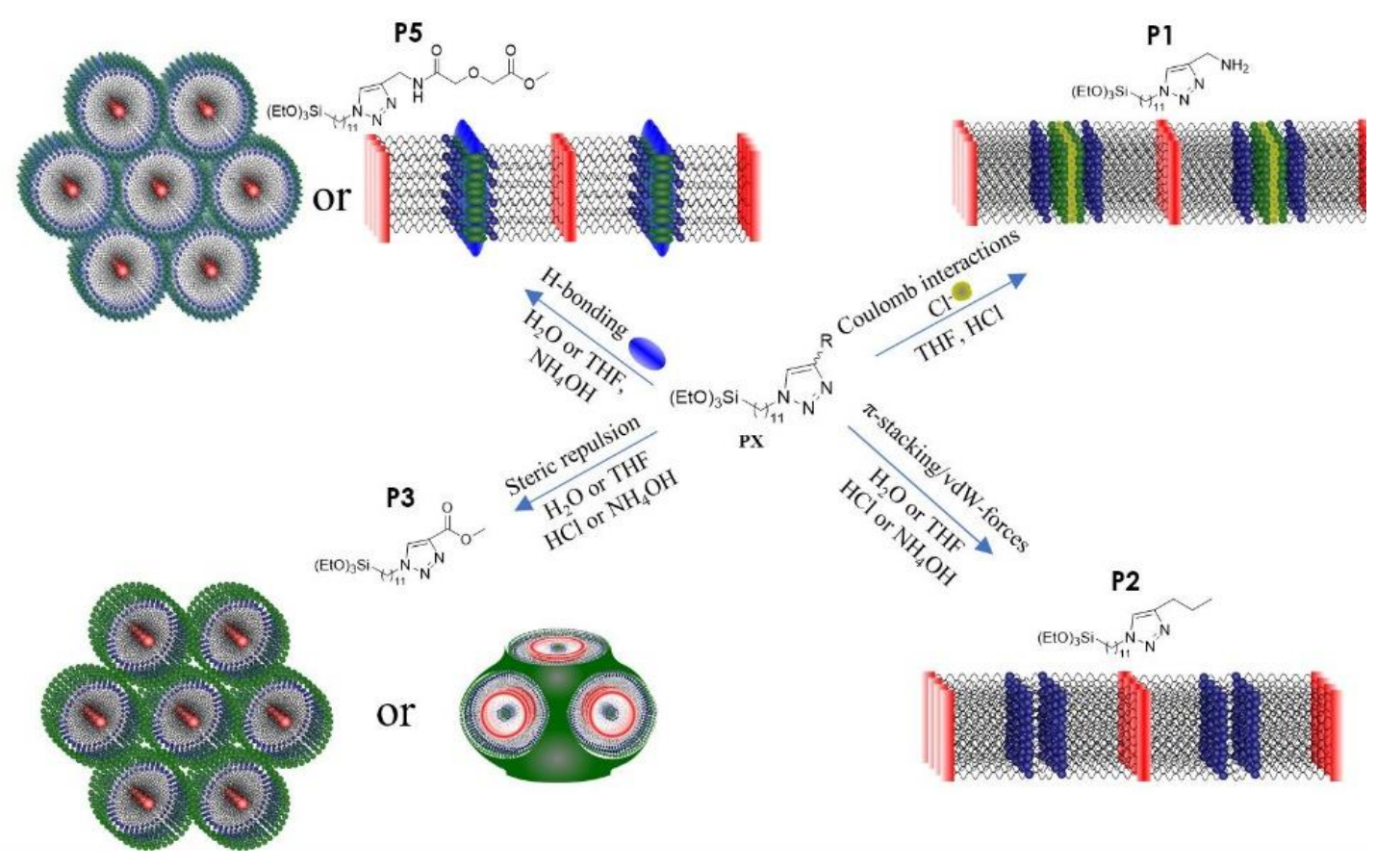

Figure 7. An overview of the discussed material morphologies and the required conditions to synthesize them.

The, thereby, induced local proximity of the silanol groups gives place to lamellae after condensation as displayed in Figure 8. This effect is less structuring in aqueous solution where a less organized structure is observed.

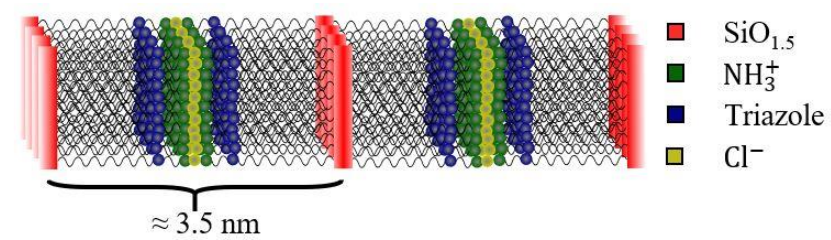

Figure 8. Schematic representation of the structural organization determined for the material P1-TA/- $\Delta$.

For P2, the silica condensation mechanism leads to more ladder-like structures under acidic conditions compared to basic conditions. Otherwise, the only potentially strong interactions are vdW forces between the precursors in solution. They have stronger effects in water than in THF. A combination of both factors allows the explanation of the lamellar characteristics in the order P2-TA $\approx$ $\mathrm{P} 2-\mathrm{TB}<\mathrm{P} 2-\mathrm{HB}<<\mathrm{P} 2-\mathrm{HA}$. The solvent nature divides the less structured (THF) from the more structured (water) lamellar morphologies by the effect of vdW forces.

The silica condensation mechanism, then, separates depending on the catalysis conditions.

In THF this effect is insignificant. In water, however, this leads to different shares of of ladder-and cage like motifs. Under acidic conditions, P2 assembles in an extended ladder-like fashion, which finally favours the formation of lamellae. This is illustrated in Figure 9.

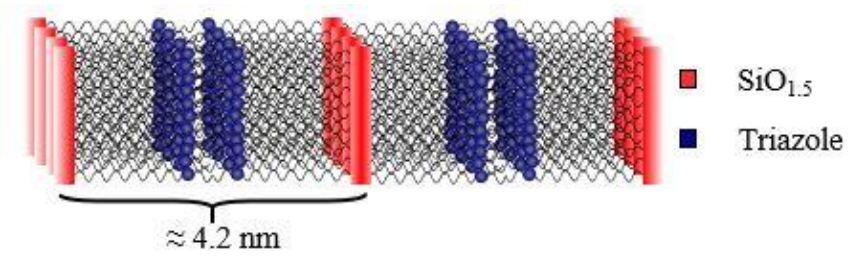

Figure 9. Schematic representation of the structural organization determined for the material P2-HA/- $\Delta$. 
The materials prepared from P3 show less impact of the catalysis conditions on the share of ladderor cage-like motifs. The effects of vdW forces between and the steric hindrance of the precursors show a stronger impact. Like observed for P2, in aqueous solution, the vdW forces of the rather hydrophobic P3 lead to highly structured morphologies. In the SAXS patterns, this is highlighted by the sharp peaks of the materials before thermal treatment.

The observed D-cubic bicontinous and hexagonal phases (see Figure 10) are induced by the branched methyl ester head group that is rigid due to its conjugation with the triazole group. During self-assembly, this steric hindrance, in analogy to surfactant-based soft matter structures, leads to structures with a mean curvature $\mathrm{k}<0$. This is energetically favourable. ${ }^{40}$ It is important to note that, for bicontinous phases, the average curvature remains close to zero. This explains the possibility a lamellar phase with the same precursor under different conditions. After the thermal treatment, only the lamellar structure, however contracted, remains observable. The curved structures are severely deformed due to the condensation of the free silanol groups.

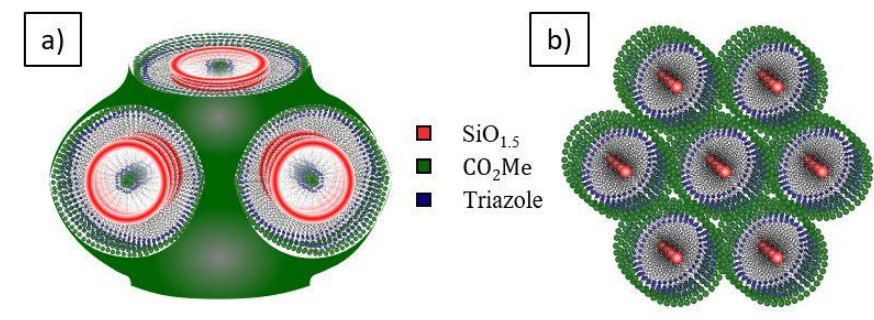

Figure 10. Schematic representation of the structural organization determined for the materials a) P3-HB and b) P3-TA.

Lastly, for the diglycolic acid derivatives (P5), hydrogen bonding is suspected to be responsible for the observed mesophases. As discussed for the scattering patterns (see Figure 5), a headgroup intercalation occurs that disappears together with the residual water after the thermal treatment. A clear link can be established using the peak shift and the hydrogen bonding evidence in the FT-IR spectra. Indeed, it can be supposed that the formation of hydrogen bonds involving residual water and the carbonyl functions is the driving force for the intercalation.

The Bragg peaks observed in the WAXS patterns support the existence of such an ordered phase. Mechanistically, the different mesophases may be caused by the persistence of hydrogen bonds in organic solvents up to high dilution in the form of agglomerates. In THF, these agglomerates would be formed by the intercalating headgroups and the limited quantity of water $(r \approx 40)$ that probably would be energetically favoured in that configuration.

Due to the geometric constraints, the silanols, then, polymerizes to form a lamellar structure (see Figure 11). In water, a large excess of hydrogen bond donors is present and, thus, the headgroup hydration is assured. This can lead to an apparent increase of the packing parameter ${ }^{8}$ and, consequently, a 2D hexagonal phase. After freeze drying, the hydrogen bonds persist thanks to residual water. Once this required residue is evaporated, the intercalation disappears as well. For P4, this mechanism, a priori, would be possible but is not observed. This might be due to the steric demand of the diethyl amide group.

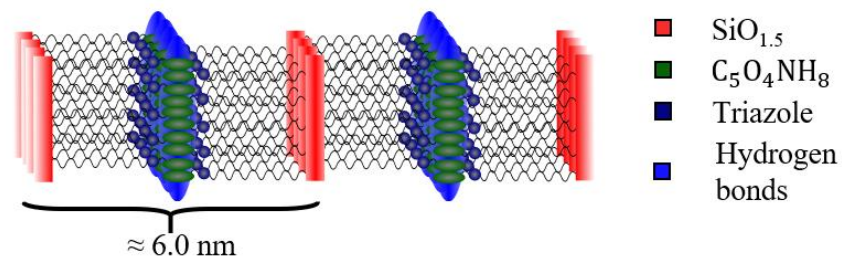

Figure 11. Schematic representation of the structural organization determined for the material P5-TB. 


\section{Conclusion}

The present work gives, for the first time, a comprehensive overview of the complex interplay between the factors that come into play during the all-in-one synthesis of SHMs. This includes strong interactions such as Coulomb interactions, vdW forces and hydrogen bonds, and steric hinderance direct the structuring that can be inherent, like the steric demand (P3), or caused by the reaction conditions, protonation of $\mathrm{P} 1$ or the $\mathrm{H}$-bonding for $\mathrm{P} 5$. Secondarily, the chemically directed connectivity of the growing Si-O-Si network nuances the obtained structure. Observations are e.g. the varying lamellar character for P2 or the structure change for P3. The factors act simultaneously and are responsible for the resulting morphology. Generally, non-lamellar structures (hexagonal, cubic) are possible in a metastable state. After the thermal treatment, the advancing condensation leads to their deformation. For further studies, a generalisation of the identified interactions can be envisaged by exploring the effects of precursor chain length or branched headgroups. Another level of complexity is possible by introducing a second silane group.

\section{Conflicts of interest}

"There are no conflicts to declare".

\section{Acknowledgements}

The authors thank Cyrielle Rey and Bruno Corso for technical support with TGA and SAXS analysis, and Diane Rébiscoul, for fruitful discussions.

\section{Notes and references}

(1) Soler-Illia, G. J. A. A.; Azzaroni, O. Multifunctional Hybrids by Combining Ordered Mesoporous Materials and Macromolecular Building Blocks. Chemical Society Reviews. The Royal Society of Chemistry January 24, 2011, pp 1107-1150. https://doi.org/10.1039/c0cs00208a.

(2) Lim, M. H.; Stein, A. Comparative Studies of Grafting and Direct Syntheses of Inorganic-Organic Hybrid Mesoporous Materials. Chem. Mater. 1999, 11 (11), 3285-3295. https://doi.org/10.1021/cm990369r.

(3) Calvo, A.; Joselevich, M.; Soler-Illia, G. J. A. A.; Williams, F. J. Chemical Reactivity of Amino-Functionalized Mesoporous Silica Thin Films Obtained by Co-Condensation and Post-Grafting Routes. Microporous Mesoporous Mater. 2009, 121 (1-3), 67-72. https://doi.org/10.1016/j.micromeso.2009.01.005.

(4) Rébiscoul, D.; Sananes Israel, S.; Tardif, S.; Larrey, V.; Ayral, A.; Rieutord, F. Impact of Silica Surface Nanoconfinement on the Microstructure of Alkoxysilane Layers Grafted by Supercritical Carbon Dioxide. J. Phys. Chem. C 2019, 123 (19), 12305-12312. https://doi.org/10.1021/acs.jpcc.9b01967.

(5) Brunel, D.; Cauvel, A.; Di Renzo, F.; Fajula, F.; Fubini, B.; Onida, B.; Garrone, E. Preferential Grafting of Alkoxysilane Coupling Agents on the Hydrophobic Portion of the Surface of Micelle-Templated Silica. New J. Chem. 2000, 24 (10), 807-813. https://doi.org/10.1039/b002945i.

(6) Yokoi, T.; Yoshitake, H.; Tatsumi, T. Synthesis of Amino-Functionalized MCM-41 via Direct Co-Condensation and Post-Synthesis Grafting Methods Using Mono-, Di- and Tri-Amino-OrganoalkoxysilanesElectronic Supplementary Information (ESI) Available: XRD Patterns of (A) x-DNN-MCM-41 and (B) x-DNNN. J. Mater. Chem. 2004, 14 (6), 951. https://doi.org/10.1039/b310576h.

(7) Besnard, R.; Cambedouzou, J.; Arrachart, G.; Diat, O.; Pellet-Rostaing, S. Self-Assembly of Condensable "BolaAmphiphiles" in Water/Tetraethoxysilane Mixtures for the Elaboration of Mesostructured Hybrid Materials. Langmuir 2013, 29 (33), 10368-10375. https://doi.org/10.1021/la401885t.

(8) Besnard, R.; Arrachart, G.; Cambedouzou, J.; Pellet-Rostaing, S. Tuning the Nanostructure of Highly Functionalized Silica Using Amphiphilic Organosilanes: Curvature Agent Effects. Langmuir 2016, 32 (18), 4624-4634. https://doi.org/10.1021/acs.langmuir.6b00589.

(9) Besnard, R.; Winkler, R.; Arrachart, G.; Cambedouzou, J.; Pellet-Rostaing, S. Ion Extraction Applications of BilayerStructured Hybrid Silicas. Mater. Chem. Front. 2018, 2 (5), 1031-1039. https://doi.org/10.1039/c8qm00022k.

(10) Besnard, R.; Arrachart, G.; Cambedouzou, J.; Pellet-Rostaing, S. Structural Study of Hybrid Silica Bilayers from "Bola-Amphiphile" Organosilane Precursors: Catalytic and Thermal Effects. RSC Adv. 2015, 5 (71), 57521-57531. https://doi.org/10.1039/C5RA06944K. 
(11) Besnard, R.; Arrachart, G.; Cambedouzou, J.; Pellet-Rostaing, S. Tuning the Morphology of Functionalized Silica Using Amphiphilic Organosilanes. J. Sol-Gel Sci. Technol. 2017, 81 (2), 452-467. https://doi.org/10.1007/s10971016-4202-1.

(12) Alauzun, J.; Mehdi, A.; Mouawia, R.; Reyé, C.; Corriu, R. J. P. Synthesis of New Lamellar Materials by Self-Assembly and Coordination Chemistry in the Solids. In Journal of Sol-Gel Science and Technology; Springer US, 2008; Vol. 46, pp 383-392. https://doi.org/10.1007/s10971-008-1710-7.

(13) Alauzun, J.; Besson, E.; Mehdi, A.; Reyé, C.; Corriu, R. J. P. Reversible Covalent Chemistry of CO2: An Opportunity for Nano-Structured Hybrid Organic-Inorganic Materials. Chem. Mater. 2008, 20 (2), 503-513.

https://doi.org/10.1021/cm701946w.

(14) Alauzun, J.; Mehdi, A.; Reyé, C.; Corriu, R. J. P. An Original Synthesis of Highly Ordered Organosilica with a High Content of Thiol Groups. Chem. Commun. 2006, No. 3, 347-349. https://doi.org/10.1039/B512537E.

(15) Voss, R.; Thomas, A.; Antonietti, M.; Ozin, G. A. Synthesis and Characterization of Highly Amine Functionalized Mesoporous Organosilicas by an "All-in-One" Approach. J. Mater. Chem. 2005, 15 (37), 4010-4014.

https://doi.org/10.1039/b507084h.

(16) Moreau, J. J. E.; Vellutini, L.; Bied, C.; Man, M. W. C. New Approach for the Organisation and the Shaping of Organo-Bridged Silicas: An Overview. J. Sol-Gel Sci. Technol. 2004, 31 (1-3), 151-156. https://doi.org/10.1023/B:JSST.0000047977.44966.53.

(17) Moreau, J. J. E.; Pichon, B. P.; Arrachart, G.; Wong Chi Man, M.; Bied, C. Nanostructuring Organo-Silicas: Combination of Intermolecular Interactions and Molecular Recognition Properties to Generate Self-Assembled Hybrids with Phenylene or Adenine *.*thymine Bridging Units. New J. Chem. 2005, 29 (5), 653.

https://doi.org/10.1039/b419376h.

(18) Winkler, R.; Pellet-Rostaing, S.; Arrachart, G. Efficient and Multi-Function Compatible Click-Reaction of Organosilanes. Tetrahedron Lett. 2020, 61 (30), 152145. https://doi.org/10.1016/j.tetlet.2020.152145.

(19) Ogata, T.; Narita, H.; Tanaka, M. Immobilization of Diglycol Amic Acid on Silica Gel for Selective Recovery of Rare Earth Elements. Chem. Lett. 2014, 43 (9), 1414-1416. https://doi.org/10.1246/cl.140446.

(20) Ogata, T.; Narita, H.; Tanaka, M. Adsorption Mechanism of Rare Earth Elements by Adsorbents with Diglycolamic Acid Ligands. Hydrometallurgy 2016, 163, 156-160. https://doi.org/10.1016/J.HYDROMET.2016.04.002.

(21) Winkler, R.; Pellet-Rostaing, S.; Arrachart, G. Selective Extraction of REEs Thanks to One-Pot Silica Hybrid Materials. Appl. Sci. 2020, 10 (21), 7558. https://doi.org/10.3390/app10217558.

(22) Al-Oweini, R.; El-Rassy, H. Synthesis and Characterization by FTIR Spectroscopy of Silica Aerogels Prepared Using Several Si(OR)4 and R"Si(OR')3 Precursors. J. Mol. Struct. 2009, 919 (1-3), 140-145.

https://doi.org/10.1016/j.molstruc.2008.08.025.

(23) Arkles, B.; Larson, G. Silicon Compounds: Silanes \& Silicones, Dr. Gupta.; Morrisville, PA, USA, 2013.

(24) Murugavel, S.; Ravikumar, C.; Jaabil, G.; Alagusundaram, P. Synthesis, Crystal Structure Analysis, Spectral Investigations (NMR, FT-IR, UV), DFT Calculations, ADMET Studies, Molecular Docking and Anticancer Activity of 2(1-Benzyl-5-Methyl-1H-1,2,3-Triazol-4-YI)-4-(2-Chlorophenyl)-6-Methoxypyridine - A Novel Poten. J. Mol. Struct. 2019, 1176, 729-742. https://doi.org/10.1016/j.molstruc.2018.09.010.

(25) Athokpam, B.; Ramesh, S. G.; McKenzie, R. H. Effect of Hydrogen Bonding on the Infrared Absorption Intensity of OH Stretch Vibrations. Chem. Phys. 2017, 488-489, 43-54. https://doi.org/10.1016/j.chemphys.2017.03.006.

(26) Philippe, L.; Sammon, C.; Lyon, S. B.; Yarwood, J. An FTIR/ATR in Situ Study of Sorption and Transport in Corrosion Protective Organic Coatings: 1. Water Sorption and the Role of Inhibitor Anions. Prog. Org. Coatings 2004, 49 (4), 302-314. https://doi.org/10.1016/J.PORGCOAT.2003.07.002.

(27) Crupi, V.; Longo, F.; Majolino, D.; Venuti, V. Vibrational Properties of Water Molecules Adsorbed in Different Zeolitic Frameworks. J. Phys. Condens. Matter 2006, 18 (15), 3563-3580. https://doi.org/10.1088/09538984/18/15/004.

(28) Katritzky, A. R.; Wang, Z.; Tsikolia, M.; Hall, C. D.; Carman, M. Benzotriazole Is Thermally More Stable than 1,2,3Triazole. Tetrahedron Lett. 2006, 47 (43), 7653-7654. https://doi.org/10.1016/J.TETLET.2006.08.021.

(29) Wong, P. T. T.; Mantsch, H. H. Pressure Effects on the Infrared Spectrum of 1,2-dipalmitoyl Phosphatidylcholine Bilayers in Water. J. Chem. Phys. 1985, 83 (7), 3268-3274. https://doi.org/10.1063/1.449185.

(30) Vaia, R. A.; Teukolsky, R. K.; Giannelis, E. P. Interlayer Structure and Molecular Environment of Alkylammonium Layered Silicates. Chem. Mater. 1994, 6 (7), 1017-1022. https://doi.org/10.1021/cm00043a025.

(31) Bantignies, J. L.; Vellutini, L.; Maurin, D.; Hermet, P.; Dieudonné, P.; Wong Chi Man, M.; Bartlett, J. R.; Bied, C.; Sauvajol, J. L.; Moreau, J. J. E. Insights into the Self-Directed Structuring of Hybrid Organic - Inorganic Silicas through Infrared Studies. J. Phys. Chem. B 2006, 110 (32), 15797-15802. https://doi.org/10.1021/jp060975r.

(32) Singh, S.; Wegmann, J.; Albert, K.; Müller, K. Variable Temperature FT-IR Studies of n-Alkyl Modified Silica Gels. J. Phys. Chem. B 2002, 106 (4), 878-888. https://doi.org/10.1021/jp012979w.

(33) Orel, B.; Ješe, R.; Vilčnik, A.; Štangar, U. L. Hydrolysis and Solvolysis of Methyltriethoxysilane Catalyzed with $\mathrm{HCl}$ or Trifluoroacetic Acid: IR Spectroscopic and Surface Energy Studies. J. Sol-Gel Sci. Technol. 2005, 34 (3), 251-265. 
https://doi.org/10.1007/s10971-005-2522-7.

(34) Lee, A. S.; Choi, S. S.; Lee, H. S.; Baek, K. Y.; Hwang, S. S. A New, Higher Yielding Synthetic Route towards Dodecaphenyl Cage Silsesquioxanes: Synthesis and Mechanistic Insights. Dalt. Trans. 2012, 41 (35), 10585-10588. https://doi.org/10.1039/c2dt30659j.

(35) Lee, A. S.; Choi, S. S.; Baek, K. Y.; Hwang, S. S. Hydrolysis Kinetics of a Sol-Gel Equilibrium Yielding Ladder-like Polysilsesquioxanes. Inorg. Chem. Commun. 2016, 73, 7-11. https://doi.org/10.1016/j.inoche.2016.09.004.

(36) Issa, A. A.; Luyt, A. S. Kinetics of Alkoxysilanes and Organoalkoxysilanes Polymerization: A Review. Polymers. Multidisciplinary Digital Publishing Institute March 21, 2019, p 537. https://doi.org/10.3390/polym11030537.

(37) Dourdain, S. Caractérisation Structurale Poreuse Mécanique de Films Minces de Silice Mésoporeuse. Influence de La Fonctionnalisation, Académie de Nantes, 2006.

(38) Skoulios, A. Organisation et Problèmes Structuraux de Copolymères Séquencés. Informations Chim. 1973, 41-46.

(39) Hyde, S. Identification of Lyotropic Liquid Crystalline Mesophases. In Handbook of Applied Surface qnd Colloid Chemistry; Holmberg, K., Ed.; Wiley, 2001; pp 300-334.

(40) Evans, D. F.; Wennerström, H. The Colloidal Domain, 2nd ed.; John Wiley \& Sons Inc: New York, 1999. 


\section{Electronic Supplementary Information for}

Tailored structuring of functionalized silsesquioxanes in an one-step approach

Robert Winkler, ${ }^{* a}$ Stéphane Pellet-Rostaing ${ }^{\text {a }}$ and Guilhem Arrachart *a

ICSM, Univ Montpellier, CEA, CNRS, ENSCM, Marcoule, France.

Material synthesis

Page S2

Figure S1. FT-IR spectra of the sample P5, $v=1800-1600 \mathrm{~cm}^{-1}$.

Page S3

Figure S2. Typical FT-IR spectrum of the residual material after the TGA analysis.

Page S4

Figure S3. TEM images of samples: a) and b) P1-TA- $\Delta$ and c) P1-TB- $\Delta$.

Page S5

Figure S4. WAXS patterns of materials P5-HB and P5-TB.

Page S6

Table S1. Fit parameters for the qualitative fit P5-TB/- $\Delta$.

Page S6 


\section{Material synthesis}

The silica hybrid material (SHM) synthesis was adapted from literature. ${ }^{1}$

In a typical synthesis, 1.5 mmoles of organosilane precursor PX were added to $50 \mathrm{~mL}$ of water and the $\mathrm{pH}$ was adjusted to basic $\left(\mathrm{NH}_{4} \mathrm{OH}, \mathrm{pH}\right.$ 9) or acidic $(\mathrm{HCl}, \mathrm{pH} 1.5)$ conditions. After stirring for two weeks in a sealed flask, the obtained precipitate was dried by freeze drying and washed with $60 \mathrm{~mL}$ $(3 \times 20 \mathrm{~mL}) \mathrm{EtOH} /$ water $(1 / 1)$ followed by $60 \mathrm{~mL}(3 \times 20 \mathrm{~mL})$ of EtOH and analysed. Afterwards, the materials were heated to $130{ }^{\circ} \mathrm{C}$ for $24 \mathrm{~h}$ to promote the condensation and washed in the same order to remove possible degradation products.

${ }^{1}$ Winkler, R.; Pellet-Rostaing, S.; Arrachart, G. Selective Extraction of REEs Thanks to One-Pot Silica Hybrid Materials. Appl. Sci. 2020, 10 (21), 7558. 


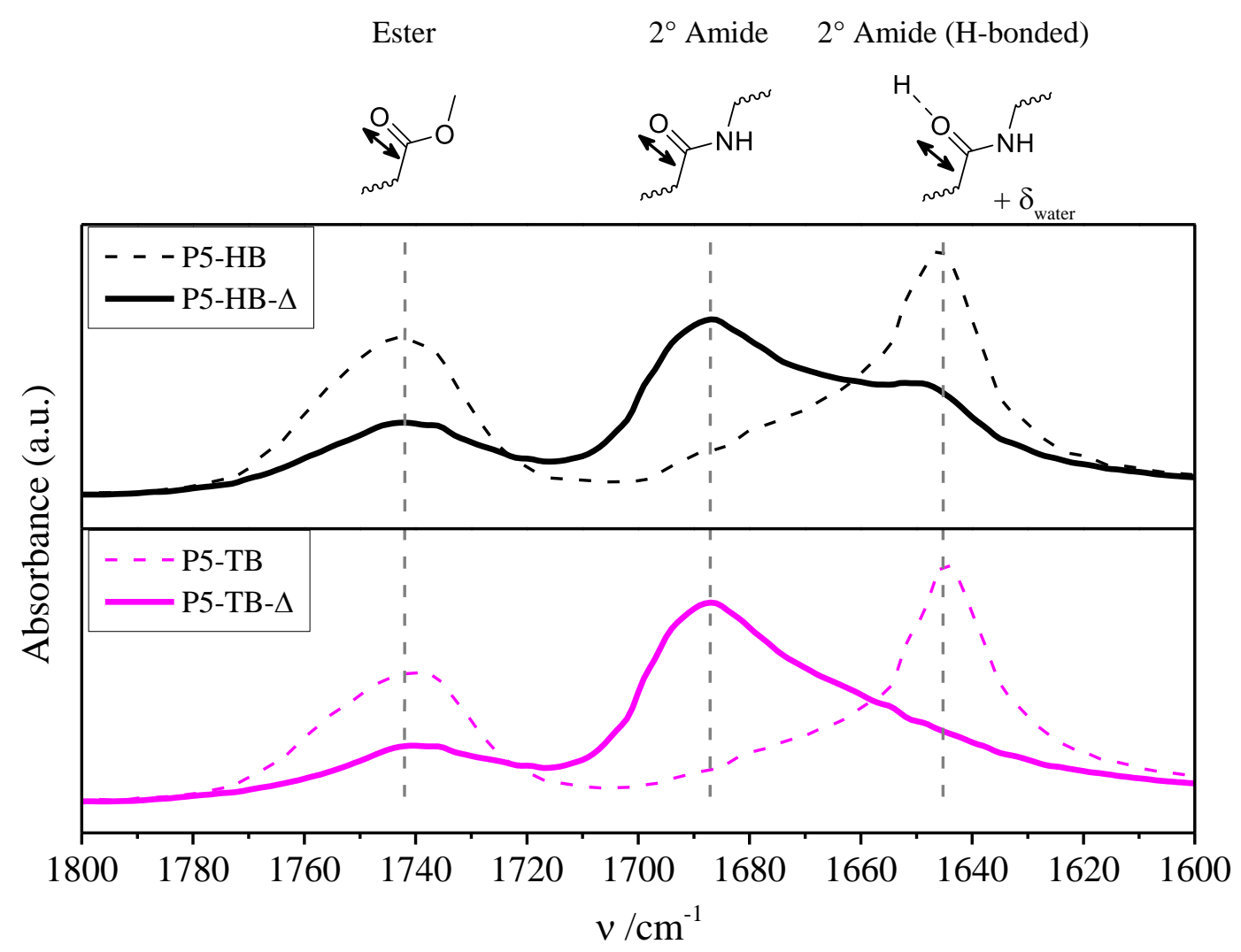

Figure S1. FT-IR spectra of the sample P5, $v=1800-1600 \mathrm{~cm}^{-1}$. 


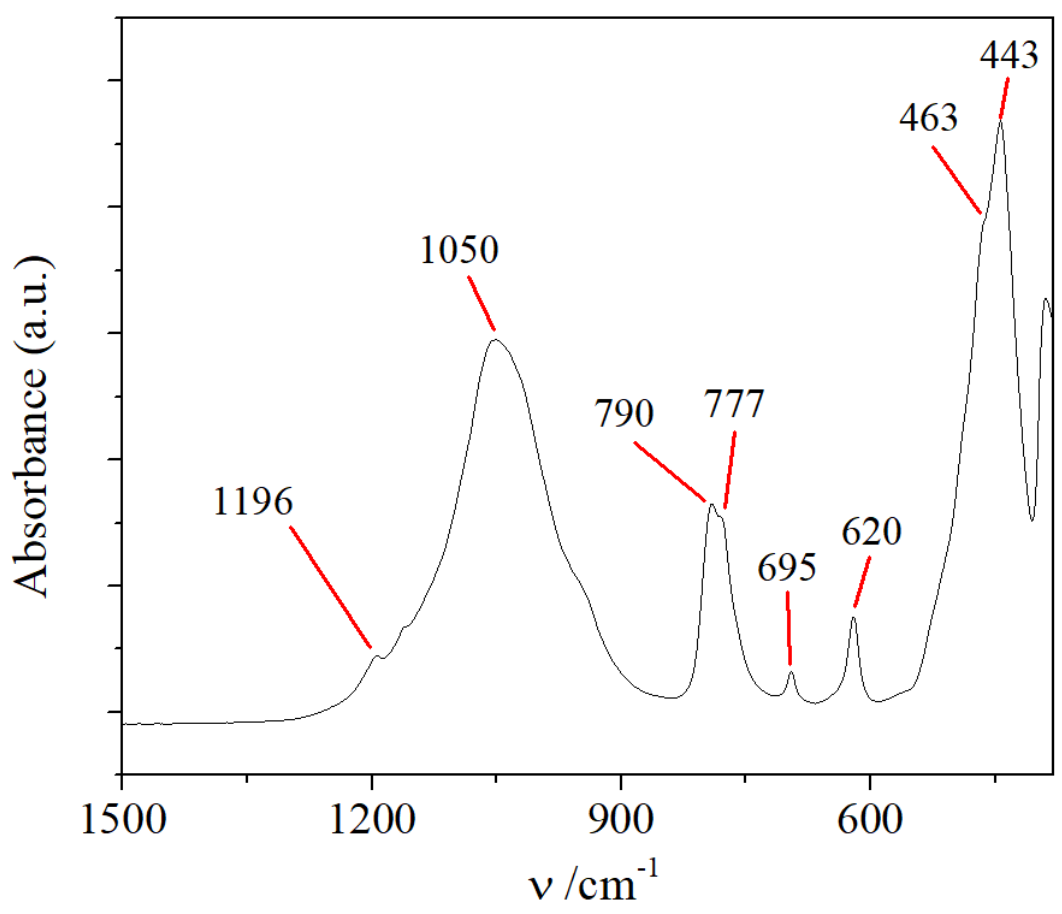

Figure S2. Typical FT-IR spectrum of the residual material after the TGA analysis.

The peaks indicate crystalline silica structures like e.g. $\beta$-cristobalite. 

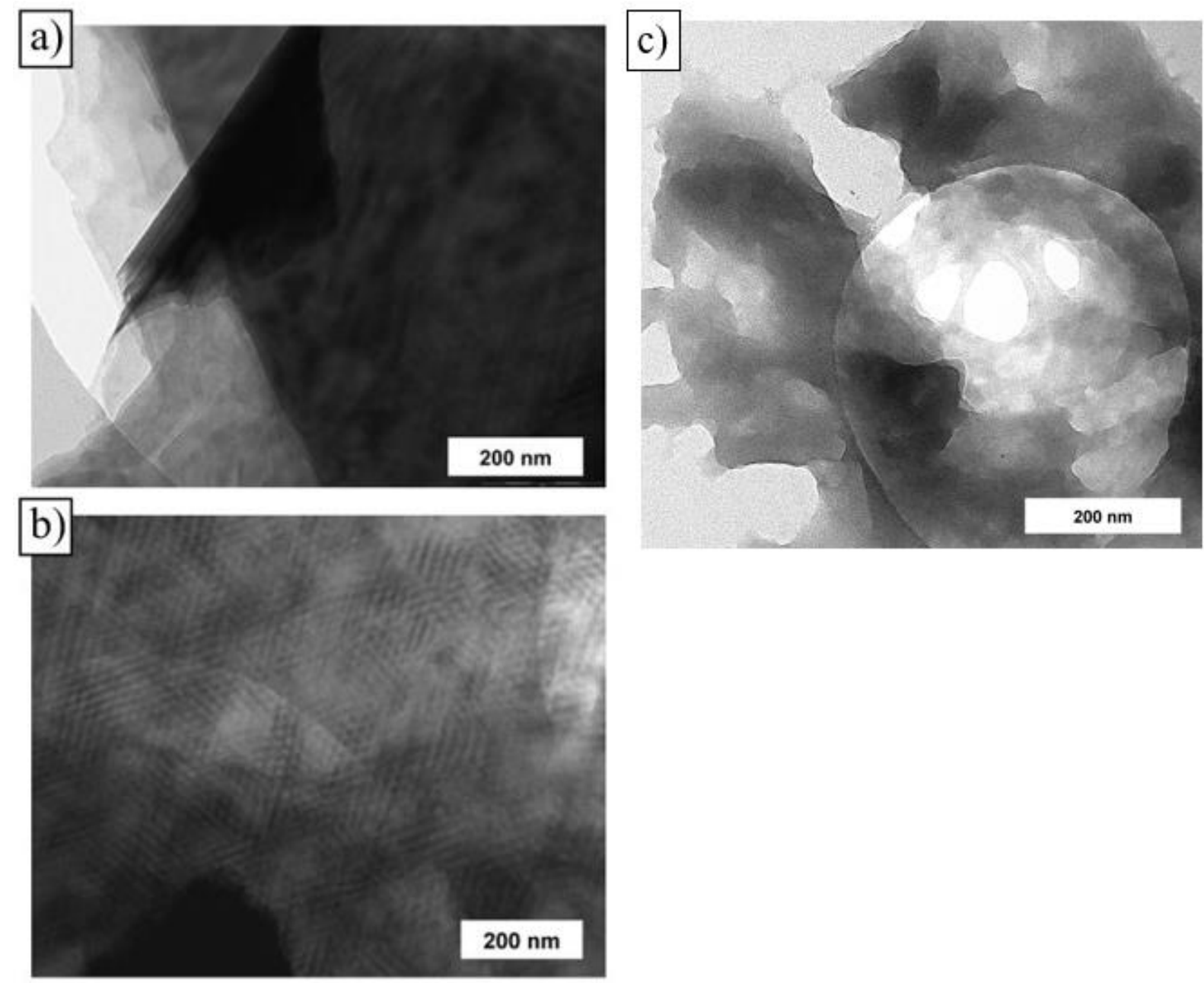

Figure S3. TEM images of samples: a) and b) P1-TA- $\Delta$ and c) P1-TB- $\Delta$.

The scale is represented on the lower right. 


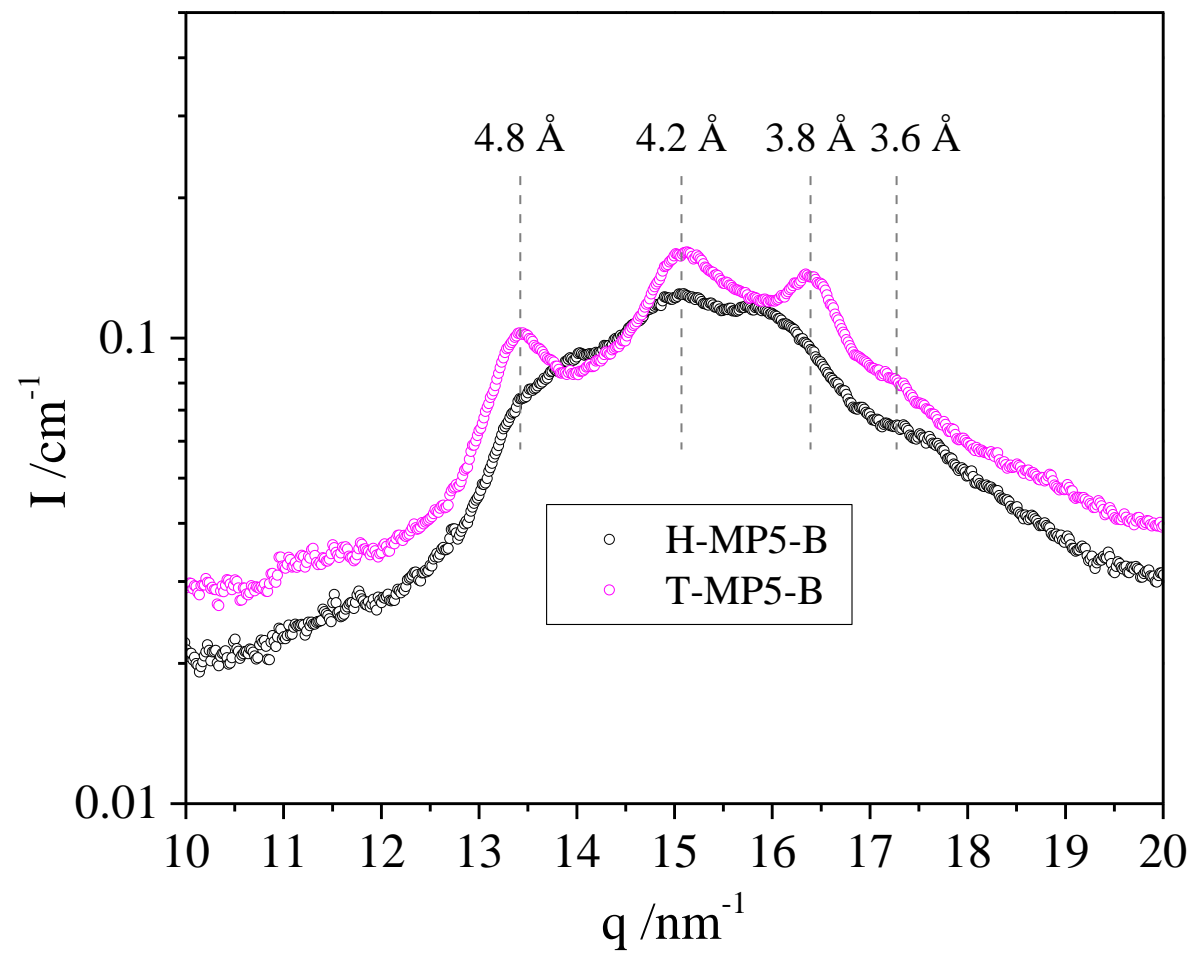

Figure S4. WAXS patterns of materials P5-HB and P5-TB.

Table S1. Fit parameters for the qualitative fit P5-TB/- $\Delta$.

\begin{tabular}{|c|c|c|c|}
\hline Parameter & Fresh material & $\begin{array}{l}\text { After thermal } \\
\text { treatment }\end{array}$ & Description \\
\hline$\rho_{\mathrm{CH} 2}$ & $0.73 \mathrm{E}-10 \mathrm{~cm}^{-2}$ & $0.73 \mathrm{E}-10 \mathrm{~cm}^{-2}$ & SLD of the alkyl chains \\
\hline$\rho_{\text {SiOx }}$ & $1.23 \mathrm{E}-10 \mathrm{~cm}^{-2}$ & $1.6 \mathrm{E}-10 \mathrm{~cm}^{-2}$ & SLD of the Si-O-Si network \\
\hline$\rho_{H G}$ & $1.04 \mathrm{E}-10 \mathrm{~cm}^{-2}$ & $0.85 \mathrm{E}-10 \mathrm{~cm}^{-2}$ & SLD of the headgroup \\
\hline$\sigma_{\text {SiOx }}$ & $0.06 \mathrm{~nm}$ & $0.06 \mathrm{~nm}$ & $\begin{array}{l}\text { FWHM of the Si-O-Si } \\
\text { network }\end{array}$ \\
\hline$\sigma_{H G}$ & $1.3 \mathrm{~nm}$ & $1.44 \mathrm{~nm}$ & FWHM of the headgroup \\
\hline$d$ & $5.95 \mathrm{~nm}$ & $6.7 \mathrm{~nm}$ & Separation distance \\
\hline
\end{tabular}

